\title{
Strict Convexity ("Continuity") of the Pressure in Lattice Systems
}

\author{
ROBERT B. GRIFFITHS and DAVID RUelle \\ Battelle Seattle Research Center, Seattle, Washington
}

Received August 27, 1971

\begin{abstract}
It is shown that the pressure is a strictly convex function of the translationally invariant interactions (under certain mild restrictions on the long-range part of these interactions) for classical and quantum lattice systems, by demonstrating that two distinct interactions can never lead to the same translationally invariant equilibrium state. This generalizes a previous result that the pressure is a continuous function of density at fixed temperature.
\end{abstract}

\section{Introduction}

The pressure $P$ has been shown to be a continuous function of the density $\varrho$, at constant temperature $T$, for certain classical [1]-[5] (p. 58), and quantum [6] systems of interacting particles in equilibrium. Since $P$ is a convex increasing function of the chemical potential $\mu$ and $\varrho=\partial P / \partial \mu$, it is evident that continuity of $P$ as a function of $\varrho$ is equivalent to the strict convexity of $P$ as a function of $\mu$ : the graph of $P(\mu)$ has no linear segments. It is then rather natural to ask whether $P$ is not also strictly convex in $T$ (and thus a continuous function of the entropy) at fixed $\mu$, or in other similar "intensive" thermodynamic variables.

In this paper we shall show that for classical and quantum lattice gases, $P$ is a strictly convex function of any linear parameter in the interaction Hamiltonian or potential energy, provided this interaction possesses translational invariance and satisfies certain other mild restrictions. The argument makes use of the relationship ([5], pp. $184 \mathrm{ff}$.) between the statistical "state" of such a system (the set of probability distributions or reduced density matrices for finite sets of lattice sites) and tangent planes to the pressure regarded as a function of the interaction $\Phi$ (further details are given below). The existence of a first-order phase transition is characterized by the possibility of at least two distinct planes tangent to $P$ for a single interaction, which is to say at least two possible states (e.g., "liquid" and "vapor"). By contrast, if $P$ were not a strictly convex function of the interactions, one could find two different interactions corresponding to the same state. We shall show that for the

13 Commun. math. Phys., Vol. 23 
lattice systems considered, different interactions necessarily lead to different states.

While our results apply to more general interactions, there is one sense in which they are inferior to previous "continuity of pressure" arguments referred to above. The latter actually establish lower bounds (depending on $\mu$ ) on the curvature of $P(\mu)$, which is to say that $P(\varrho)$ satisfies a Lipschitz condition or, in physical terms, there is a finite lower bound to the compressibility. Our argument, by contrast, does not rule out the possibility that $P$ as a function of a suitable intensive parameter might have occasional points of vanishing curvature.

\section{Generalities and Statement of Results}

We define a (translation invariant) interaction for a classical lattice gas to be a real function $\Phi$ of finite subsets of $\mathbb{Z}^{v}$ satisfying the conditions

(I 1) $\Phi(\phi)=0$;

(I 2) $\|\Phi\|=\sum_{X \ni 0}|\Phi(X)|<+\infty$;

(I 3) $\Phi(X+x)=\Phi(X)$ for all $x \in \mathbb{Z}^{v}$ (translation invariance).

These interactions form a Banach space $B$ with respect to the norm $\|\cdot\|$. A real function $P$ (the pressure, essentially) is defined on $B$ by

$$
P(\Phi)=\lim _{\Lambda \rightarrow \infty}|\Lambda|^{-1} \log \sum_{X \subset A} \exp \left[-\sum_{Y \subset X} \Phi(Y)\right],
$$

where $|\Lambda|$ is the number of points in the finite set $\Lambda$, which tends to infinity in some sense, for instance a parallelipiped with all sides tending to infinity. It is known (see [5], Ch. 2) that the limit (2.1) exists, and that $P$ is a continuous convex function on $B$, in fact ${ }^{1}$

and

$$
\mid P(\Phi)-P(\Psi) \leqq\|\Phi-\Psi\|
$$

$$
P\left(\frac{1}{2} \Phi+\frac{1}{2} \Psi\right) \leqq \frac{1}{2} P(\Phi)+\frac{1}{2} P(\Psi) .
$$

We shall show that, if $\Phi \neq \Psi$,

$$
P\left(\frac{1}{2} \Phi+\frac{1}{2} \Psi\right)<\frac{1}{2} P(\Phi)+\frac{1}{2} P(\Psi),
$$

i.e., $P$ is strictly convex.

The strict convexity of the pressure can also be proven for quantum lattice systems. Let $\mathscr{K}$ be a finite dimensional complex Hilbert space and $\mathscr{K}_{x}$ a copy of $\mathscr{K}$ for every $x \in \mathbb{Z}^{v}$. For every finite $X \in \mathbb{Z}^{v}$ we write

$$
\mathscr{K}_{X}=\bigotimes_{x \in X} \mathscr{K}_{x},
$$

1 These results would hold also if (I 2) were replaced by the less restrictive condition $\|\Phi\|=\sum_{X \ni 0}|\Phi(X)| /|X|<+\infty$, but we shall use (I 2$)$ for the proof of strict convexity of $P$. 
and let $\mathcal{O}_{X}$ be the algebra of all bounded operators on $\mathscr{K}_{X}$. If $Y \subset X$, we have $\mathscr{K}_{X}=\mathscr{K}_{Y} \otimes \mathscr{K}_{X \backslash Y}$ and we can identify any $A \in \mathcal{O}_{Y}$ with an element of $\mathcal{O}_{X}$ by $A \rightarrow A \otimes \mathbb{1}$. In this manner all the $\mathcal{O}_{X}$ are subalgebras of a big algebra $\tilde{\mathcal{O}}$. We define now a (translation invariant) interaction to be a function $\Phi$ of finite subsets of $\mathbb{Z}^{v}$ satisfying the conditions

(QI 0) $\Phi(X)$ is a self-adjoint element of $\mathcal{O}_{X}$;

(QI 1) For $Y \subset X$ (finite) we let $\operatorname{Tr}_{Y}$ be the trace on $\mathscr{K}_{Y}$ and thus a partial trace on $\mathscr{K}_{X}$ : for $A \in \mathcal{O}_{Y}, B \in \mathcal{O}_{X \backslash Y}$,

$$
\operatorname{Tr}_{Y} A \otimes B=\left(\operatorname{Tr}_{Y} A\right) B \text {. }
$$

Then for any $Y \subset X, \operatorname{Tr}_{Y} \Phi(X)=0$;

(QI 2) For some fixed $\xi>0$,

$$
\|\Phi\|=\sum_{X \ni 0}\|\Phi(X)\| \exp (\xi|X|)<\infty ;
$$

(QI 3) $\Phi(X+x)=\tau_{x} \Phi(X)$ for all $x \in \mathbb{Z}^{v}$, where $\tau_{x}$ is the canonical isomorphism $\mathcal{O}_{x} \rightarrow \mathcal{O}_{X+x}$ (translation invariance).

As for the classical lattice gas, the interactions form a Banach space $B$ with respect to the norm $\|\cdot\|$ and a real function $P$ is defined on $B$ by

$$
P(\Phi)=\lim _{\Lambda \rightarrow \infty}|\Lambda|^{-1} \log \operatorname{Tr}_{\Lambda} \exp \left[-\sum_{Y \subset \Lambda} \Phi(Y)\right] .
$$

It is known (see [5], Ch. 2) that the limit (2.5) exists and that $P$ satisfies (2.2) and $(2.3)^{2}$.

Theorem 1. The function $P$ defined by (2.1) (resp. (2.5)) is strictly convex on the Banach space $B$ of interactions of a classical lattice gas (resp. quantum lattice system) as defined above.

Let us say that a continuous linear functional $\alpha$ on $B$ is tangent to $P$ over $\Phi$ if

$$
P(\Phi+\Psi) \geqq P(\Phi)+\alpha(\Psi)
$$

for all $\Psi \in B$. Because of (2.2) and (2.3), there is at least one tangent linear functional over each $\Phi \in B$.

Proposition 2. A linear functional $\alpha$ cannot be tangent to $P$ over two different points $\Phi$ and $\Phi^{\prime}$.

Theorem 1 is clearly equivalent to Proposition 2 . We now sketch the proof of Proposition 2 (the details appear in Section III for classical lattice gases, in Section IV for quantum lattice systems). It is known that a linear functional tangent to $P$ corresponds uniquely to a state satisfying

\footnotetext{
${ }^{2}$ As before, we remark that these results would still hold if $(Q$ I 2$)$ were replaced by the condition $\sum_{X \ni 0}\|\Phi(X)\| /|X|<\infty$, but $(Q$ I 2$)$ will be used in Theorem 1 .
} 
the variational principle of equilibrium statistical mechanics. Such a state satisfies the equilibrium equations of Dobrushin in the classical case, and is a KMS state in the quantum case. It remains therefore to show that a state can satisfy the equilibrium equations or the KMS condition for only one interaction. In the classical case this is fairly easy to see, and in the quantum case it follows from the Tomita-Takesaki theory.

\section{The Case of Classical Lattice Gases}

A state of a classical lattice gas is a probability measure $\sigma$ on the set $\mathscr{P}\left(\mathbb{Z}^{\nu}\right)$ of subsets of $\mathbb{Z}^{\nu}$, or, equivalently, a positive linear functional on the space of continuous functions on $\mathscr{P}\left(\mathbb{Z}^{v}\right)$. (The topology of $\mathscr{P}\left(\mathbb{Z}^{v}\right)$ is determined by the condition that subsets $X_{n}$ converges to $X$ when for any finite set $\Lambda, X_{n} \cap \Lambda$ agrees with $X \cap \Lambda$ for $n$ sufficiently large; with this topology, $\mathscr{P}\left(\mathbb{Z}^{v}\right)$ is compact.) Given a tangent plane to $P$ at $\Phi$, that is a linear functional $\alpha$ on $B$ satisfying (2.6), it follows from [5], Section 7.5, that there is a unique state $\sigma$ invariant under lattice translations and such that

$$
\alpha(\Psi)=-\int A_{\Psi}(Y) \sigma(d Y),
$$

where $A_{\Psi}$ is a continuous function on $P\left(\mathbb{Z}^{\nu}\right)$ defined by

$$
A_{\Psi}(Y)=\sum_{0 \in S \subset Y} \Psi(S) /|S|
$$

with the sum over finite subsets of $Y$. Furthermore, this state satisfies equilibrium equations (Lanford and Ruelle [7]; see also Dobrushin [8]) of the form

$$
\sigma_{\Lambda}(X, d Y)=f_{\Lambda}(X, Y) \sigma_{\Lambda}(\phi, d Y),
$$

where $\Lambda$ is a finite set with $X \subset \Lambda$ the configuration of particles (the set of occupied sites) in $\Lambda, Y \subset \mathbb{Z}^{v} \backslash \Lambda$,

$$
f_{\Lambda}(X, Y)=\exp \left[\underset{S \subset X \cup Y: S \cap X \neq \phi}{-\sum_{S(S)}} \Phi,\right.
$$

and $\sigma_{\Lambda}(X, d Y)$ is a measure on the subsets of $\mathbb{Z}^{v} \backslash \Lambda$ defined as follows: if $\mathscr{L} \subset \mathscr{P}\left(\mathbb{Z}^{v} \backslash \Lambda\right)$ is measurable, then

$$
\sigma_{\Lambda}(X, \mathscr{L})=\sigma\{W \cup X: W \in \mathscr{L}\} .
$$

The probability of finding a given configuration $X$ in $\Lambda$ is just

$$
\sigma_{\Lambda}(X)=\int \sigma_{\Lambda}(X, d Y) .
$$


Of course, the sum over all $X \subset \Lambda$ of $\sigma_{\Lambda}(X)$ is 1 ; this fact together with the bound

$$
e^{-|A| \cdot\|\Phi\|} \leqq\left|f_{\Lambda}(X, Y)\right| \leqq e^{|\Lambda| \cdot\|\Phi\|},
$$

and the equation (3.3) imply that $\sigma_{\Lambda}(X)$ is strictly positive for all $X$ (including $X=\phi$ ) in $\Lambda$, and hence ratios of such probabilities (see (3.7) below) make sense.

We wish to show that given the state $\sigma$ we can deduce unambiguously the interaction $\Phi$. The procedure is most transparent in the case of an interaction of finite range: $\Phi(X)$ vanishes whenever the maximum

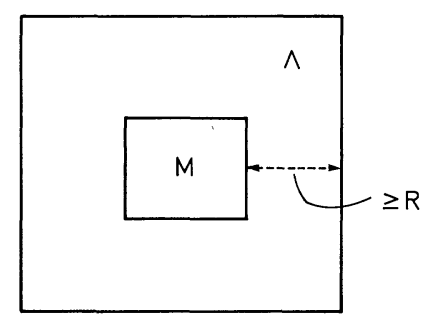

Fig. 1.

distance between a pair of points in $X$ exceeds a constant, $R$. We suppose, for the moment, that $X$ is a subset of a set $M \subset \Lambda$, and $\Lambda$ is large enough so that the distance from a point inside $M$ to a point outside $\Lambda$ exceeds $R$ (see Fig. 1). It is then apparent from its definition that $f_{A}(X, Y)$ is independent of $Y$, and thus, combining (3.3) and (3.5),

$$
\sigma_{\Lambda}(X) / \sigma_{\Lambda}(\phi)=\exp \left[-\sum_{S \subset X} \Phi(S)\right] .
$$

The state determines the left, and hence the right, side of (3.7) for every finite $X$, and consequently also, by induction, $\Phi(X)$.

For interactions not restricted to finite range we note that for a fixed $M$ and given $\varepsilon>0$, it is always possible, because of (I2), to choose $\Lambda$ large enough (but still finite) so that

$$
\sum_{\substack{X \subset M \\ X \neq \phi}} \sum_{\substack{Y \subset \mathbb{Z}^{v} \backslash \Lambda \\ Y \neq \phi}}|\Phi(X \cup Y)|<\varepsilon .
$$

Thus, although $f_{A}(X, Y)$ depends on $Y$, its variation with $Y$ (for fixed $X \subset M)$ is slight, and the ratio $\sigma_{\Lambda}(X) / \sigma_{\Lambda}(\phi)$ is, within a factor of $e^{ \pm \varepsilon}$, again given by the right side of (3.7). By letting $\Lambda$ tend to infinity in a suitable sense we have $\varepsilon \rightarrow 0$, and the state again determines uniquely $\Phi(X)$. This completes the proof of Proposition 1 for classical lattice gas. 


\section{The Case of Quantum Lattice Systems}

Let $\mathcal{O}$ be obtained by completing the algebra $\tilde{\mathcal{O}}=\bigcup_{\Lambda} \mathcal{O}_{\Lambda}$ with respect to its norm. Then $\mathcal{O}$ is a $C^{*}$-algebra. Because of (QI 2), every interaction $\Phi \in B$ determines a one-parameter group of automorphisms of $\mathcal{O}$ :

$$
\bar{\tau}_{t} A=\lim _{\Lambda \rightarrow \infty} \exp \left[i t \sum_{X \subset \boldsymbol{A}} \Phi(X)\right] A \exp \left[-i t \sum_{X \subset \boldsymbol{A}} \Phi(X)\right] .
$$

See Robinson [9], and [5], Section 7.6.

A state of a quantum lattice system is a positive linear functional $\sigma$ on the $C^{*}$-algebra $\mathcal{O}$ such that $\sigma(1)=1$. The state $\sigma$ is an equilibrium state if it satisfies the KMS condition: if $A, B \in \mathcal{O}$, there exists a bounded continuous function $F$ on $\{z: 0 \leqq \operatorname{Im} z \leqq 1\}$, analytic for $0<\operatorname{Im} z<1$ and such that for all real $t$

$$
\sigma\left(B \cdot \bar{\tau}_{t} A\right)=F(t), \quad \sigma\left(\bar{\tau}_{t} A \cdot B\right)=F(t+i) .
$$

Now let $\alpha$ be a linear functional on $B$ satisfying (2.6). It follows from [5], Section 7.5, and from Lanford and Robinson [10], that there is a unique state $\sigma$ invariant under lattice translations and such that

$$
\alpha(\Psi)=-\sigma\left(A_{\Psi}\right),
$$

where $A_{\Psi} \in \mathcal{O}$ is defined by

$$
A_{\Psi}=\sum_{X \ni 0} \frac{\Psi(X)}{|X|} .
$$

To conclude the proof of Proposition 1 we shall now show that if $\sigma$ and $\sigma^{\prime}$ are equilibrium states for the interactions $\Phi$ and $\Phi^{\prime}$ respectively, then $\Phi \neq \Phi^{\prime}$ implies $\sigma \neq \sigma^{\prime}$. Let $\bar{\tau}$ and $\bar{\tau}^{\prime}$ be the one-parameter groups of automorphisms of $\mathcal{O}$ corresponding to the interactions $\Phi$ and $\Phi^{\prime}$, and with respect to which the states $\sigma$ and $\sigma^{\prime}$ are, respectively, KMS states. It is known that if $\sigma=\sigma^{\prime}$, then $\bar{\tau}=\bar{\tau}^{\prime}$ (see Takesaki [11], Ch. 13). Therefore if $A \in \mathcal{O}_{\Delta}$ for some finite $\Delta$

$$
\sum_{X: X \cap \Delta \neq \phi}[A, \Phi(X)]=i \frac{d}{d t} \bar{\tau}_{t} A=i \frac{d}{d t} \bar{\tau}_{t}^{\prime} A=\sum_{X: X \cap \Delta \neq \phi}\left[A, \Phi^{\prime}(X)\right] .
$$

It remains thus to prove that

implies $\Psi=0$.

$$
\sum_{X: X \cap \Delta=\phi}[A, \Psi(X)]=0
$$

For $B \subset \mathcal{O}_{A}$ and $\Delta C A$ we define

$$
\pi_{\Delta} B=\left(\operatorname{Tr}_{\Lambda \backslash \Delta} 1\right)^{-1}{ }^{-1} \operatorname{Tr}_{\Lambda \backslash \Delta} B .
$$


Then $\pi_{\Delta}: \mathcal{O}_{\Lambda} \rightarrow \mathcal{O}_{\Delta}$ has norm 1 and extends by continuity to a map $\mathcal{O} \rightarrow \mathcal{O}_{\Delta}$ again denoted by $\pi_{\Delta}$. Applying $\pi_{\Delta}$ to (4.2) we find

$$
\sum_{X \subset \Delta}[A, \Psi(X)]=0 .
$$

We prove now that $\Psi(X)=0$ by induction on $|X|$. Since $\Psi(\phi)=0$, it suffices to show that $[A, \Psi(\Delta)]=0$ for all $A \in \mathcal{O}_{A}$ implies $\Psi(\Delta)=0$, but this is obvious because a traceless multiple of the identity must vanish.

Acknowledgments. It is a pleasure to acknowledge helpful discussions with J. Ginibre and O. E. Lanford III.

\section{References}

1. Ruelle, D.: Helv. Phys. Acta 36, 183 (1963).

2. Dobrushin, R.L., Minlos, R.A.: Teor. Veroyat. Ee Primen. 12, 596 (1967) [English translation: Theory Probab. Its Appl. 12, 535 (1967)].

3. Ginibre, J.: Phys. Letters 24 A, 223 (1967).

4. Ruelle, D.: Commun. math. Phys. 18, 127 (1970).

5. - Statistical mechanics: Rigorous results. New York: W. A. Benjamin Inc 1969.

6. Ginibre, J.: Phys. Rev. Letters 24, 1473 (1970).

7. Lanford III, O.E., Ruelle, D.: Commun. math. Phys. 13, 194 (1969).

8. Dobrushin, R.L.: Funkts. Analiz. Prilozh. 2, No. 4, (1968) p. 31 [English translation: Functional Anal. Applic. 2, 292 (1968)].

9. Robinson, D. W.: Commun. math. Phys. 7, 337 (1968).

10. Lanford III, O.E., Robinson, D. W.: Commun. math. Phys. 9, 327 (1968).

11. Takesaki,M.: Tomita's theory of modular Hilbert algebras and its applications. Berlin, Heidelberg, New York: Springer 1970.

David Ruelle

I.H.E.S., F-91 Bures-sur-Yvette, France

Robert B. Griffiths

Physics Department,

Carnegie-Mellon University,

Pittsburgh, Pennsylvania 15213, USA 